\title{
On the Exponential Integrability of Conjugate Functions
}

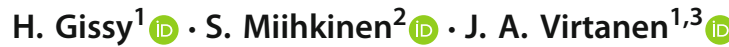

Received: 25 December 2020 / Revised: 30 July 2021 / Accepted: 9 August 2021 /

Published online: 18 October 2021

(C) The Author(s) 2021

\section{Abstract}

We relate the exponential integrability of the conjugate function $\tilde{f}$ to the size of the gap in the essential range of $f$. Our main result complements a related theorem of Zygmund.

Keywords Exponential integrability $\cdot$ Conjugate function $\cdot$ Hilbert transform $\cdot$ Outer functions

Mathematics Subject Classification 42A50

\section{Introduction}

We denote by $L^{p}$ the usual Lebesgue spaces of functions on the unit circle $\mathbb{T}$ with norm $\|\cdot\|_{p}$. Given $f \in L^{1}$, let $u$ be the Poisson integral of $f$ and denote by $\tilde{u}$ the harmonic conjugate function of $u$, normalized so that $\tilde{u}(0)=0$. Then $\tilde{u}(z)$ has nontangential limit $\tilde{f}(\theta)$ almost everywhere on $\mathbb{T}$ and we call $\tilde{f}$ the conjugate function of $f$. Alternatively,

Communicated by Alexey Karapetyants.

Gissy was supported by a University of Reading doctoral grant. Miihkinen was supported by Emil Aaltonen Foundation. Virtanen was supported in part by Engineering and Physical Sciences Research Council grant EP/T008636/1.

$凶 \quad$ J. A. Virtanen

j.a.virtanen@reading.ac.uk; jani.virtanen@helsinki.fi

H. Gissy

h.m.h.gissy@pgr.reading.ac.uk

S. Miihkinen

santeri.miihkinen@kau.se

1 Department of Mathematics, University of Reading, Reading, England

2 Department of Mathematics and Computer Science, Karlstad University, Karlstad, Sweden

3 Department of Mathematics, University of Helsinki, Helsinki, Finland 
the conjugate function $\tilde{f}$ can be defined as the principal value integral

$$
\tilde{f}(\theta)=\lim _{\epsilon \rightarrow 0} \frac{1}{2 \pi} \int_{|\theta-\varphi|>\epsilon} \cot \left(\frac{\theta-\varphi}{2}\right) f(\varphi) d \varphi
$$

for almost every $\theta$. For further details and references, see Sect. 2.1 below.

The linear mapping $f \mapsto \tilde{f}$ is referred to as the conjugation operator. If $f$ is a trigonometric polynomial $\sum_{n=-N}^{N} a_{n} e^{i n \theta}$, then $\tilde{f}$ is a trigonometric polynomial of the same degree

$$
\tilde{f}(\theta)=\sum_{n=-N}^{N}-i \operatorname{sgn}(n) a_{n} e^{i n \theta},
$$

where $-i$ sgn is the Fourier multiplier associated with the conjugation operator.

When $1<p<\infty$, according to a famous theorem of M. Riesz, there is a constant $C_{p}$ such that

$$
\|\tilde{f}\|_{p} \leq C_{p}\|f\|_{p}
$$

for all $f \in L^{p}$. In addition, although $f \in L^{\infty}$ does not imply that $\tilde{f} \in L^{\infty}$ (see, e.g. [4]), the Hilbert transform still has very strong boundedness properties as can be seen in the following theorem, due to Zygmund [19].

Theorem A (Zygmund) For $f \in L^{\infty}$ with $\|f\|_{\infty} \leq \pi / 2$ and $\lambda<1$, there is $a$ constant $C_{\lambda}$ such that

$$
\frac{1}{2 \pi} \int_{0}^{2 \pi} e^{\lambda|\tilde{f}|}<C_{\lambda}
$$

and if $f$ is continuous on $\mathbb{T}$, then

$$
\frac{1}{2 \pi} \int_{0}^{2 \pi} e^{\lambda|\tilde{f}|}<\infty
$$

for all $\lambda<\infty$.

For the proof, see Corollary III.2.6 of [4]. It follows that

$$
f=f_{1}+f_{2},\left\|f_{1}\right\|_{\infty}<\pi / 2, f_{2} \in C(\mathbb{T}) \Longrightarrow \exp (\tilde{f}) \in L^{1},
$$

where $C(\mathbb{T})$ stands for the space of continuous functions on $\mathbb{T}$.

Let $E$ be a measurable subset of $\mathbb{T}$ and define

$$
\rho_{E}(z)=2 \chi_{E}(z)-1= \begin{cases}1, & z \in E \\ -1, & z \notin \mathbb{T} \backslash E .\end{cases}
$$


The Lebesgue measure of $E$ is denoted by $|E|$. We note that the condition $\left\|f_{1}\right\|_{\infty}<$ $\pi / 2$ above is optimal as seen by considering an interval $E=[a, b] \subset(0,2 \pi)$ and showing that

$$
\exp \left(\frac{\pi}{2} \tilde{\rho}_{E}(t)\right)=\exp \left(-\log \left|\sin \frac{t-b}{2}\right|+\log \left|\sin \frac{t-a}{2}\right|\right)=\left|\frac{\sin \frac{t-a}{2}}{\sin \frac{t-b}{2}}\right|
$$

which is not integrable in any neighborhood of $b$. More generally, it follows from (1.6) and Theorem III.2.7 of [4] that $\exp \left(\frac{\pi}{2} \tilde{\rho}_{E}\right) \notin L^{1}$ whenever $E$ is a measurable subset of $\mathbb{T}$ with $0<|E|<2 \pi$.

Notice that the conditions of (1.4) imply that if the function $f$ is real valued and has jumps, then the size of each jump is strictly less than $\pi$, while the size of each jump of $\frac{\pi}{2} \rho_{E}$ is exactly $\pi$ and $\exp \left(\frac{\pi}{2} \tilde{\rho}_{E}\right) \notin L^{1}$ with $E$ as above. Motivated by the study of the Fredholm properties of Toeplitz operators, Shargorodsky [11] proved that if $g$ is real valued and $\inf \mathcal{R}(g)>\pi / 2$, where $\mathcal{R}(g)$ stands for the essential range of $g$, then $\exp \left(\widetilde{g \rho_{E}}\right)$ is not integrable. These observations lead to the question of whether

$$
f \in L^{\infty} \text { and } f \geq \pi / 2 \text { a.e. } \Longrightarrow \exp \left(\widetilde{f \rho_{E}}\right) \notin L^{1}
$$

Our main result answers this in the affirmative. Indeed, we give an elementary proof of the following result in Sect. 3.

Theorem 1 Suppose that $f \in L^{\infty}$ with $f \geq \pi / 2$ almost everywhere and $0<|E|<$ $2 \pi$. Then there is a positive constant $C$ such that

$$
\left|E_{\lambda}\right|=\left|\left\{t: \widetilde{f \rho_{E}}(t)>\lambda\right\}\right| \geq C e^{-\lambda}
$$

for all $\lambda \geq 0$. In particular,

$$
\exp \left(\widetilde{f \rho_{E}}\right) \notin L^{1}
$$

Remark 1 In the preceding theorem, the conditions on $E$ and that $f \geq \pi / 2$ almost everywhere are optimal—see Remark 3.

Remark 2 Notice that [11] contains the following local non-integrability result. Suppose that $0<|E|<2 \pi$ and $\gamma \subset \mathbb{T}$ is an arc intersecting both $E$ and $\mathbb{T} \backslash E$ in sets of positive measure and at least one of these sets is not an arc (modulo sets of measure zero). If $g \in L^{\infty}$ is real valued and $\inf \mathcal{R}(g)>\pi / 2$, then $\exp \left(\widetilde{g \rho_{E}}\right)$ is not integrable on $\gamma$.

Notice that Theorem 1 generalizes the preceding result for $\gamma=\mathbb{T}$. However, it remains an open problem whether our conclusion can be strengthened to that of [11] for the other $\operatorname{arcs} \gamma \neq \mathbb{T}$ if we only assume that $g \geq \pi / 2$ almost everywhere.

Previously in $[9,15]$, sufficient conditions for exponential integrability of $\tilde{f}$ were obtained in terms of the modulus of continuity of $f$ in $L^{p}$. In addition to these results 
and other intrinsic interest $[4,7,18]$, the integrability of the exponential of conjugate functions plays an important role in the spectral theory of Toeplitz and related operators [10-12], scalar Riemann-Hilbert problems [13,14,17] and their applications.

\section{Preliminaries}

As indicated in the introduction, our approach is elementary and based on classical results of complex analysis which are briefly discussed in this section.

\subsection{Poisson Integrals}

For $f \in L^{1}$, denote by $P[f]$ the Poisson integral of $f$, that is,

$$
P[f](z)=\frac{1}{2 \pi} \int_{0}^{2 \pi} P_{z}(\theta) f(\theta) d \theta \quad(z \in \mathbb{D}),
$$

where the Poisson kernel $P_{z}$ is defined by

$$
P_{z}(\theta)=\operatorname{Re} \frac{e^{i \theta}+z}{e^{i \theta}-z}
$$

Recall that $P[f]$ is harmonic in $\mathbb{D}$ and if the function $f$ is continuous at $e^{i \theta}$, then

$$
\lim _{z \rightarrow e^{i \theta}} P[f](z)=f(\theta)
$$

(see Theorem I.1.3 of [5]). To deal with discontinuities at $e^{i \theta}$, define a cone $\Gamma_{\alpha}$ by

$$
\Gamma_{\alpha}\left(e^{i \theta}\right)=\left\{z \in \mathbb{D}:\left|z-e^{i \theta}\right|<\alpha(1-|z|)\right\}
$$

for each $\alpha<1$, and recall that a function $\varphi: \mathbb{D} \rightarrow \mathbb{C}$ is said to have nontangential $\operatorname{limit} \varphi^{*}\left(e^{i \theta}\right)$ at $e^{i \theta}$ if

$$
\lim _{\Gamma_{\alpha}\left(e^{i \theta}\right) \ni z \rightarrow e^{i \theta}} \varphi(z)=\varphi^{*}\left(e^{i \theta}\right)
$$

for every $\alpha<1$. Now, for any $f \in L^{1}$, if $u=P[f]$, then $u^{*}=f$ almost everywhere by Fatou's theorem.

Define

$$
X_{f}(z)=\frac{1}{2 \pi} \int_{0}^{2 \pi} \frac{e^{i \theta}+z}{e^{i \theta}-z} f(\theta) d \theta \quad(z \in \mathbb{D})
$$

Then

$$
u(z)+i \tilde{u}(z)=X_{f}(z)=\frac{1}{2 \pi} \int_{0}^{2 \pi} \frac{e^{i \theta}+z}{e^{i \theta}-z} f(\theta) d \theta
$$


for $z \in \mathbb{D}$, where $u=P[f]$ and $\tilde{u}$ is the harmonic conjugate function of $u$ normalized so that $\tilde{u}(0)=0$. By Fatou's theorem and Lemma III.1.1 of [4],

$$
X_{f}^{*}(\theta)=f(\theta)+i \tilde{f}(\theta)
$$

for almost every $\theta$. For the integral representation of $\tilde{f}$ given in (1.1), see Lemma III.1.2 of [4]. In particular, it follows that the principal value in (1.1) exists almost everywhere. If $f \in L^{\infty}$, then

$$
\left|\operatorname{Re} X_{f}(z)\right|=|u(z)|<\|f\|_{\infty} .
$$

\subsection{Harmonic and Subharmonic Functions}

In one of the key steps of the proof of the main theorem, we consider the Dirichlet problem of finding a unique bounded harmonic function on a simply connected domain $\Omega$ with prescribed boundary values.

If $g$ is a continuous real-valued function on $\partial \Omega$, the Dirichlet problem of finding the bounded harmonic function $u: \Omega \rightarrow \mathbb{R}$ such that $u=g$ on $\partial \Omega$ can be solved using the Poisson integral and the Riemann mapping theorem, which reduces the problem to the well-known case of the unit disk (see, e.g., [5]).

However, in our case, since the boundary functions are discontinuous (see Lemma 1 below), the following more general result is needed.

Theorem B Let $\Omega$ be a simply connected domain and let $g$ be a piecewise continuous function on $\partial \Omega$ with a finite number of discontinuities of the first kind at $\xi_{1}, \ldots \xi_{k}$. Then there is at most one bounded harmonic function $h$ on $\Omega$ such that $h=g$ on $\partial \Omega \backslash\left\{\xi_{1}, \ldots, \xi_{k}\right\}$.

If such a bounded harmonic function $h$ exists, then

$$
\inf _{\zeta \in \partial \Omega \backslash\left\{\xi_{1}, \ldots, \xi_{k}\right\}} h(\zeta) \leq h(z) \leq \sup _{\zeta \in \partial \Omega \backslash\left\{\xi_{1}, \ldots, \xi_{k}\right\}} h(\zeta)
$$

for all $z \in \Omega$.

For the proof of the preceding result, see Theorems 5 and 6 of Sect. 42 of [8].

Regarding the values of a harmonic function in a domain $\Omega$ (a nonempty open connected set), we recall the maximum principle (Theorem 1.8 of [2]):

Theorem C Suppose $\Omega$ is a domain, $u$ is real-valued and harmonic on $\Omega$, and $u$ has a maximum or a minimum in $\Omega$. Then $u$ is constant.

Lemmas 1 and 2 below are utilized in key steps of the proof of the main result.

Lemma 1 Suppose that $f \in L^{\infty}$ with $f \geq \frac{\pi}{2}$ almost everywhere. Let $\lambda \geq 0$ and

$$
G_{\lambda}=\{|\operatorname{Re} z|<\pi / 2\} \cup\left\{z:|\operatorname{Re} z|<\|f\|_{\infty} \text { and } \operatorname{Im} z>\lambda+1\right\}
$$


be the domain in Fig. 1 and put $L=\partial G_{\lambda} \cap\{\operatorname{Im} z>\lambda+1\}$. Then there exists a unique bounded harmonic function $v_{\lambda}$ on $G_{\lambda}$ with the boundary values

$$
v_{\lambda}(z)= \begin{cases}1, & z \in L, \\ 0, & z \in \partial G_{\lambda} \backslash\left(L \cup\left\{ \pm\|f\|_{\infty}+i(\lambda+1)\right\}\right) .\end{cases}
$$

Proof Let $\tau$ be a conformal map of $G_{\lambda}$ onto the unit disk $\mathbb{D}$. Then each straight-line piece of the boundary $\partial G_{\lambda}$ is mapped to an arc on the circle $\mathbb{T}$ (see Theorem II.3.4' of [6]). Now consider the Poisson integral of the function that equals 1 on the arcs to which $\tau$ maps $L$ and 0 on the complementary arcs. The composition of the Poisson integral with $\tau$ gives the desired harmonic function. Uniqueness and the bounds

$$
0<v_{\lambda}(z)<1 \text { for } z \in G_{\lambda}
$$

follow from Theorems B and C.

The following characterization of subharmonic functions is also needed:

Theorem $\mathrm{D}$ Let $\mathrm{u}$ be a function on $\mathbb{D}$ and suppose it satisfies the following conditions:

(i) $\infty \leq u<\infty, u \not \equiv-\infty$,

(ii) $u$ is upper semi-continuous in $\mathbb{D}$,

(iii) for each $z_{0} \in \mathbb{D}$, there is an $r_{0}$ such that $D\left(z_{0}, r_{0}\right) \subset \mathbb{D}$ and

$$
u\left(z_{0}\right) \leq \frac{1}{2 \pi} \int_{0}^{2 \pi} u\left(z_{0}+r e^{i \theta}\right) d \theta
$$

for all $0 \leq r \leq r_{0}$.

Then $u$ is subharmonic in $\mathbb{D}$.

For the proof of the preceding result, see Theorem II.13 of [16].

Lemma 2 Let $\lambda, f, G_{\lambda}$, and $v_{\lambda}$ be as in Lemma 1 and $E \subset \mathbb{T}$ be a measurable set with $0<|E|<2 \pi$. Define

$$
X(z)=\frac{1}{2 \pi} \int_{0}^{2 \pi} \frac{e^{i \theta}+z}{e^{i \theta}-z}\left(f \rho_{E}\right)(\theta) d \theta \quad(z \in \mathbb{D}),
$$

where $\rho_{E}$ is defined in (1.5). Let $\Omega_{\lambda}=X^{-1}\left(G_{\lambda}\right)$ and define $H_{\lambda}: \mathbb{D} \rightarrow \mathbb{C}$ by

$$
H_{\lambda}(z)= \begin{cases}v_{\lambda} \circ X, & z \in \Omega_{\lambda} \\ 0, & z \in \mathbb{D} \backslash \Omega_{\lambda} .\end{cases}
$$

Then $H_{\lambda}$ is continuous and subharmonic with $0 \leq H_{\lambda}<1$. 


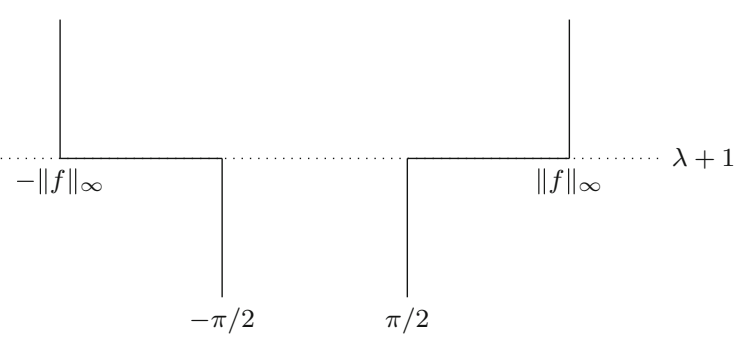

Fig. 1 The open set $G_{\lambda}$

Proof First, if $z \in \partial \Omega_{\lambda} \cap \mathbb{D}$, then $X(z) \notin G_{\lambda}$ but $X(z) \in \overline{G_{\lambda} \cap X(\mathbb{D})}$, and hence $X(z) \in \partial G_{\lambda}$. Further, since $|\operatorname{Re} X(z)|<\|f\|_{\infty}$ and $z \in \mathbb{D}$,

$$
X(z) \in\left\{x+i y:|x|<\|f\|_{\infty}, y \leq \lambda+1\right\}
$$

and so $v_{\lambda}(X(z))=0$ according to (2.7)). If $z_{k} \rightarrow z$ in $\Omega_{\lambda}$, then, using the continuity of $v_{\lambda}$ up to the point $X(z), v_{\lambda}\left(X\left(z_{k}\right)\right) \rightarrow 0$, which implies that $H_{\lambda}$ is continuous on $\mathbb{D}$. That $0 \leq H_{\lambda}<1$ follows from $0<v_{\lambda}<1$ (see (2.8)) and the definition of the function $H_{\lambda}$.

The local mean value inequality in (2.9) holds for each point of $\Omega_{\lambda}$ because $H_{\lambda}=$ $v_{\lambda} \circ X$ is harmonic in $\Omega_{\lambda}$, and it holds for each point of $\mathbb{D} \backslash \Omega_{\lambda}$ because $H_{\lambda} \geq 0$ equals zero there. Thus, by Theorem $\mathrm{D}$, the function $H_{\lambda}$ is subharmonic in $\mathbb{D}$.

\section{Proof of Theorem 1}

Suppose that $f \in L^{\infty}$ with $f \geq \pi / 2$ almost everywhere and $0<|E|<2 \pi$. Let

$$
X(z)=\frac{1}{2 \pi} \int_{0}^{2 \pi} \frac{e^{i \theta}+z}{e^{i \theta}-z}\left(f \rho_{E}\right)(\theta) d \theta \quad(z \in \mathbb{D}),
$$

where $\rho_{E}$ is defined in (1.5). Then

$$
X^{*}=f \rho_{E}+\widetilde{i f \rho_{E}}
$$

almost everywhere according to (2.5).

The proof of (1.8) consists of several steps.

Step 1 Since $\operatorname{Re} X^{*}=f \rho_{E} \geq \pi / 2$ almost everywhere on $E$ and $\operatorname{Re} X^{*} \leq-\pi / 2$ almost everywhere on $\mathbb{T} \backslash E$, there is a point $w \in \mathbb{D}$ such that $|\operatorname{Re} X(w)|<\pi / 2$.

Step 2 Let the open set $G_{\lambda}$ (see Fig. 1) and $L=\partial G_{\lambda} \cap\{\operatorname{Im} z>\lambda+1\}$ be defined as in Lemma 1. 
Then Lemma 1 asserts that there exists a unique bounded harmonic function $v_{\lambda}$ on $G_{\lambda}$ with the boundary values

$$
v_{\lambda}(z)= \begin{cases}1, & z \in L \\ 0, & z \in \partial G_{\lambda} \backslash\left(L \cup\left\{ \pm\|f\|_{\infty}+i(\lambda+1)\right\}\right) .\end{cases}
$$

Step 3 Let $\Omega_{\lambda}=X^{-1}\left(G_{\lambda}\right)$. Then, according to Lemma 2, the function $H_{\lambda}: \mathbb{D} \rightarrow \mathbb{C}$ defined by

$$
H_{\lambda}(z)= \begin{cases}v_{\lambda} \circ X, & z \in \Omega_{\lambda} \\ 0, & z \in \mathbb{D} \backslash \Omega_{\lambda}\end{cases}
$$

is continuous and subharmonic with $0 \leq H_{\lambda}<1$.

Step 4 There is a $C>0$, independent of $\lambda$, such that

$$
\left|E_{\lambda}\right| \geq C\left(v_{\lambda} \circ X\right)(w)
$$

where $E_{\lambda}$ is defined by (1.8) and $w$ is defined in Step 1 . Since $H_{\lambda}$ is bounded, it trivially has a harmonic majorant, and hence, by Theorem I.6.7 of [4],

$$
H_{\lambda}(z) \leq \lim _{r \rightarrow 1} \frac{1}{2 \pi} \int_{0}^{2 \pi} P_{z}(\theta) H_{\lambda}\left(r e^{i \theta}\right) d \theta
$$

for $z \in \mathbb{D}$. It follows from (2.5) that, for almost every $\theta \in[0,2 \pi] \backslash E_{\lambda}$,

$$
\left|\operatorname{Re} X^{*}(\theta)\right|=|f(\theta)| \geq \pi / 2 \text { and } \operatorname{Im} X^{*}(\theta) \leq \lambda .
$$

Therefore, using the definition of $H_{\lambda}$ and the properties of $v_{\lambda}$,

$$
\lim _{r \rightarrow 1} H_{\lambda}\left(r e^{i \theta}\right)=v_{\lambda}\left(X^{*}(\theta)\right)=0 .
$$

Now, by (3.2) and Lebesgue's dominated convergence theorem,

$$
\begin{aligned}
H_{\lambda}(w) & \leq \frac{1}{2 \pi} \int_{E_{\lambda}} P_{w}(\theta) d \theta+\frac{1}{2 \pi} \int_{[0,2 \pi] \backslash E_{\lambda}} P_{w}(\theta) \lim _{r \rightarrow 1} H_{\lambda}\left(r e^{i \theta}\right) d \theta \\
& =\frac{1}{2 \pi} \int_{0}^{2 \pi} P_{w}(\theta) \chi_{E_{\lambda}}(\theta) d \theta \leq C \int_{0}^{2 \pi} \chi_{E_{\lambda}}(\theta) d \theta=C\left|E_{\lambda}\right|
\end{aligned}
$$

where the constant is independent of $\lambda$.

Step 5 It is difficult to obtain the desired estimate $\left(v_{\lambda} \circ X\right)(w) \geq C e^{-\lambda}$ directly. Instead we estimate $v_{\lambda}$ from below by another harmonic function $g_{\lambda}$, defined on a vertical strip, which allows us to compute $g_{\lambda}$ explicitly in the next step.

Let $g_{\lambda}$ be the bounded harmonic function in the strip $S=\{|\operatorname{Re} z|<\pi / 2\}$ with the boundary values 


$$
g_{\lambda}( \pm \pi / 2+i y)= \begin{cases}1, & y>\lambda+2 \\ 0, & y<\lambda+2\end{cases}
$$

Notice that $\lim _{y \rightarrow \infty} v_{0}( \pm \pi / 2+i y)=1$ (which can be verified using a conformal map of $\Omega_{0}$ onto $\mathbb{D}$ ). Therefore, since $v_{0}$ is continuous, Theorem $\mathrm{C}$ implies that there is a positive constant $C$ such that

$$
v_{0}( \pm \pi / 2+i y) \geq C \geq C g_{0}( \pm \pi / 2+i y)
$$

for all $y>2$. Clearly $v_{0}( \pm \pi / 2+i y) \geq 0=C g_{0}( \pm \pi / 2+i y)$ for all $y<2$. Thus, by Theorem $\mathrm{B}, v_{0}(z)-C g_{0}(z) \geq 0$ for all $z \in S$, and so for $\lambda>0$ and $z \in S$,

$$
v_{\lambda}(z)=v_{0}(z-i \lambda) \geq C g_{0}(z-i \lambda)=C g_{\lambda}(z)
$$

Consequently, by (3.1),

$$
\left|E_{\lambda}\right| \geq C\left(g_{\lambda} \circ X\right)(w)
$$

where the constant $C$ is independent of $\lambda$.

Step 6 Let $\tau=x+i y=X(w)$, so $|x|<\pi / 2$ and $y \in \mathbb{R}$. We show that there is a constant $C>0$, independent of $\lambda$, such that $g_{\lambda}(\tau) \geq C e^{-\lambda}$, which completes the proof.

$$
F(z)=\tan \frac{1}{2} z=-i\left(\frac{e^{\frac{1}{2} i z}-e^{-\frac{1}{2} i z}}{e^{\frac{1}{2} i z}+e^{-\frac{1}{2} i z}}\right)
$$

defines a conformal mapping from $S$ onto $\mathbb{D}$ with $F(i b)=i \tanh \left(\frac{1}{2} b\right) \rightarrow \pm i$, as $b \rightarrow \pm \infty$. Notice that

$$
F^{-1}(z)=2 \arctan z=i \log \frac{1-i z}{1+i z}
$$

for $z \in \mathbb{D}$. Thus, using the mapping $z \mapsto-i z$, we see that

$$
z \mapsto i \log \frac{1-\tan \frac{z}{2}}{1+\tan \frac{z}{2}}=-i \log \frac{1+\tan \frac{z}{2}}{1-\tan \frac{z}{2}}
$$

maps $S$ conformally onto itself with $\pm \frac{\pi}{2} \mapsto(0, \mp \infty)$ and $(0, \infty) \mapsto \frac{\pi}{2}$. Therefore,

$$
\begin{aligned}
g_{\lambda}(z) & =\frac{1}{2}+\frac{1}{\pi} \operatorname{Re}\left(-i \log \frac{1+\tan \frac{z-i(\lambda+2)}{2}}{1-\tan \frac{z-i(\lambda+2)}{2}}\right) \\
& =\frac{1}{2}+\frac{1}{\pi} \arg \frac{1+\tan \frac{z-i(\lambda+2)}{2}}{1-\tan \frac{z-i(\lambda+2)}{2}} .
\end{aligned}
$$


To evaluate $g_{\lambda}$ at $\tau=x+i y$, write $\tau_{\lambda}=\frac{\tau-i(\lambda+2)}{2}$ and notice that

$$
\begin{aligned}
g_{\lambda}(\tau) & =\frac{1}{2}+\frac{1}{\pi} \arg \frac{1+\tan \tau_{\lambda}}{1-\tan \tau_{\lambda}}=\frac{1}{2}+\frac{1}{\pi} \arg \tan \left(\tau_{\lambda}+\pi / 4\right) \\
& =\frac{1}{2}+\frac{1}{\pi} \arctan \frac{\sinh (y-(\lambda+2))}{\sin (x+\pi / 2)}=\frac{1}{2}-\frac{1}{\pi} \arctan \frac{\sinh (\lambda+2-y)}{\cos x}
\end{aligned}
$$

using the formula $\arg \tan (a+i b)=\arctan \frac{\sinh 2 b}{\sin 2 a}$ and the fact that $\arctan$ is odd. Observe also that the expression for $g_{\lambda}(\tau)$ is valid in $S$, but not necessarily on $\partial S$ and the first expression for $g_{\lambda}(z)$ is valid in $\bar{S}$. We first established the expression for $g_{\lambda}(z)$ with the desired boundary behavior on $\partial S$ and then considered only the behavior inside $S$, where the expressions for $g_{\lambda}(z)$ and $g_{\lambda}(\tau)$ coincide. Since $\arctan a=\frac{\pi}{2}-\arctan a^{-1}$ for $a>0$, we have, for $\lambda+2>y$,

$$
g_{\lambda}(\tau)=\frac{1}{\pi} \arctan \frac{\cos x}{\sinh (\lambda+2-y)},
$$

which gives the estimate. This completes the proof of (1.8).

It remains to prove (1.9). As in Sect. 4 of Chapter I in [4], consider the distribution function

$$
m(\lambda)=\left|\left\{t: \exp \left(\widetilde{f \rho_{E}}(t)\right)>\lambda\right\}\right|
$$

for $\lambda>0$. Notice that for $\lambda \geq 1$,

$$
m(\lambda)=\left|E_{\log \lambda}\right|
$$

(see (1.8) for the definition of $E_{\log \lambda}$ ), and so by Lemma I.4.1 in [4],

$$
\left.\int \exp \widetilde{f \rho_{E}}(\theta)\right) d \theta=\int_{0}^{\infty} m(\lambda) d \lambda \geq \int_{1}^{\infty}\left|E_{\log \lambda}\right| d \lambda .
$$

It remains to combine (1.8) with (3.3).

\section{Further Remarks}

In this section we provide remarks and examples related to Theorem 1 . We show first that the conditions in the theorem are optimal.

Remark 3 In Theorem 1, (i) the condition on $E$ is optimal, and (ii) the condition that $f \geq \pi / 2$ almost everywhere is optimal.

Proof (i) Let $E=[0,2 \pi]$ and $f=\pi / 2$ on $E$. Then $f \rho_{E}=f$ and so trivially $e^{\widetilde{f \rho_{E}}} \in L^{1}$ by (1.3). 
Further, by Corollary III.1.8 of [7],

$$
\left|E_{\lambda}\right|=\left|\left\{t: \widetilde{f \rho_{E}}\left(e^{i t}\right) \mid>\lambda\right\}\right|=\left|\left\{t:\left|\tilde{\chi}_{E}\left(e^{i t}\right)\right|>2 \lambda / \pi\right\}\right|<10 \pi e^{-2 \lambda},
$$

which implies that there is no constant $C$ for which (1.8) holds in this case.

(ii) Suppose that $\pi / 4<f<\pi / 2$ on an interval $I=(a, b) \subset(0,2 \pi)$ and $f=\pi / 2$ on $[0,2 \pi] \backslash I$. Let $E=[0, a+\delta] \cup[b-\delta, 2 \pi]$ for some small $\delta>0$. Then $\exp \left(\widetilde{f \rho_{E}}\right)$ is integrable by Zygmund's Theorem A because the gap in the essential range of $f \rho_{E}$ is strictly less than $\pi$. Consequently, the condition is optimal for (1.9), and it must also be optimal for (1.8) because it was used to prove (1.9).

Remark 4 In addition to the example in the previous proof, there are functions $f$ which are not constant and still satisfy $\left|E_{\lambda}\right| \lesssim e^{-\lambda}$ as in (4.1). Indeed, let $0<|E|<2 \pi$ and let $f$ be Hölder with $f(\theta)=\pi / 2$ for all $\theta \in \partial E$. If $g=(f-\pi / 2) \rho_{E}$, then it is not difficult to see that $g$ is Hölder and hence $\tilde{g}$ is Hölder. Also,

$$
\widetilde{f \rho_{E}}-\tilde{g}=\frac{\pi}{2} \tilde{\rho}_{E}
$$

Thus, by Exercise VI.18 of [4],

$$
\left|\left\{t: \widetilde{f \rho_{E}}(t)-\tilde{g}(t) \mid>\lambda\right\}\right|=\left|\left\{t:\left|\frac{\pi}{2} \tilde{\rho}_{E}(t)\right|>\lambda\right\}\right| \lesssim e^{-\lambda},
$$

which implies that $\left|E_{\lambda}\right|=\left|\left\{t: \widetilde{f \rho_{E}}(t) \mid>\lambda\right\}\right| \lesssim e^{-\lambda+\|\tilde{g}\|_{\infty}} \leq C e^{-\lambda}$ for some constant $C$.

In fact, (0.2) of [18], when $\psi=\widetilde{f \rho_{E}}=\tilde{g}+\frac{\pi}{2} \tilde{\rho}_{E}$, implies the stronger result

$$
\sup _{I} \frac{\left|\left\{t \in I:\left|\psi(t)-\psi_{I}\right|>\lambda\right\}\right|}{|I|} \lesssim e^{-\lambda}
$$

Notice that the converse is not true, however, that is, (4.2) does not imply that

$$
\psi=u+\tilde{v}, \quad u \in L^{\infty},\|v\|_{\infty} \leq \pi / 2
$$

(see Wolff's counterexample on page 52 of [18]).

Open Problem 1 In the preceding remark, when $\psi=\frac{1}{2} \log |H|$ with $H$ univalent and zero free, Baernstein [3,18] posed a question of whether (4.2) implies (4.3). This seems to be still open.

Our next example concerns outer functions from the theory of Hardy spaces. Recall that an outer function is a function $G$ on the unit disk which can be written in the form

$$
G(z)=\alpha \exp \left(\frac{1}{2 \pi} \int_{0}^{2 \pi} \frac{e^{i t}+z}{e^{i t}-z} \log \varphi(t) d t\right), \quad \alpha \in \mathbb{C}, z \in \mathbb{D},
$$

where $|\alpha|=1$ and $\varphi$ is a positive measurable function on $\mathbb{T}$ such that $\log \varphi \in L^{1}$. Similarly to (2.4), 


$$
G(z)=e^{u(z)+i v(z)}, \quad z \in \mathbb{D}
$$

where $u$ is the Poisson integral of $\log \varphi$ and $v$ is the harmonic conjugate function of $u$ so that $e^{i v(0)}=\alpha$.

Let $0<p<\infty$ and let $f$ be analytic in $\mathbb{D}$. Then the function $f$ is in the Hardy space $H^{p}$ if

$$
\sup _{r<1} \int_{0}^{2 \pi}\left|f\left(r e^{i t}\right)\right|^{p} d t<\infty
$$

Notice that $G \in H^{p}$ if and only if $\varphi \in L^{p}$ (see, e.g., Sect. II.4 of [4]). We can now use Theorem 1 to determine when certain outer functions are not in $H^{p}$ as shown in the following example.

Example 1 Given a real-valued function $f$ in $L^{\infty}$, define

$$
\Phi_{f}(z)=\exp \left(\frac{i}{4 \pi} \int_{0}^{2 \pi} \frac{e^{i t}+z}{e^{i t}-z} f(t) d t\right), \quad|z|<1 .
$$

Using (4.5), it is easy to see that $\Phi_{f}$ and its inverse $\Phi_{f}^{-1}$ are both outer functions. Denote by $\mathcal{R}(f)$ the essential range of $f$ as before. Let

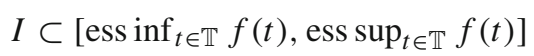

be an interval such that $I \cap \mathcal{R}(f)=\emptyset$ and $|I| \geq \frac{2 \pi}{p}$. Then $\Phi_{f}, \Phi_{f}^{-1} \notin H^{p}$. In particular, $\Phi_{f}^{ \pm 1} \notin H^{2}$ if $\mathcal{R}(f)$ has a gap of length $\geq \pi$.

To see this, notice first that (similarly to (2.5))

$$
\left(\Phi_{f}^{ \pm 1}\right)^{*}(t)=\exp \left(\mp \frac{1}{2}(\tilde{f}(t)-i f(t))\right),
$$

so $\left|\left(\Phi_{f}^{ \pm 1}\right)^{*}(t)\right|=e^{\mp \frac{1}{2} \tilde{f}(t)}$. Since $\Phi_{f}$ is an outer function, it follows from Theorem 1 that $\Phi_{f}, \Phi_{f}^{-1} \notin H^{p}$ if $\frac{p}{2}|I| \geq \pi$.

To illustrate the effect of jumps in relation to exponential integrability (see Example 2), the following lemma will be needed. Its proof is included for completeness because we have not found it in the literature.

Lemma 3 For $0<x<2 \pi$, let

$$
g(x)=\sum_{n=2}^{\infty} \frac{\cos n x}{n \log n} .
$$

Then

$$
g(x)=\log \log \frac{1}{x}+B+O\left(\left(\log \log \frac{1}{x}\right)^{1 / 3}\left(\log \frac{1}{x}\right)^{-2 / 3}\right)
$$

as $x \rightarrow 0+$, where $B$ is a positive constant. 
Proof Define $B_{x}(y)=\sum_{2 \leq n \leq y} \cos n x$. Then, for $0<x<\pi$ and $y>2,\left|B_{x}(y)\right| \leq$ $\frac{\pi}{x}+1$. Therefore, integrating by parts,

$\sum_{n=N}^{M} \frac{\cos n x}{n \log n}=\int_{N}^{M} \frac{d B_{x}(y)}{y \log y}=\frac{B_{x}(M)}{M \log M}-\frac{B_{x}(N)}{N \log N}+\int_{N}^{M} \frac{B_{x}(y)(\log y+1)}{y^{2}|\log y|^{2}} d y$,

and so

$$
\begin{aligned}
\left|\sum_{n=N}^{\infty} \frac{\cos n x}{n \log n}\right| & \leq \frac{\frac{\pi}{x}+1}{N \log N}+\left(\frac{\pi}{x}+1\right) \int_{N}^{\infty} \frac{\log y+1}{y^{2} \log ^{2} y} d y \\
& \leq 2 \frac{\frac{\pi}{x}+1}{N \log N} \leq \frac{4 \pi}{x N \log N}
\end{aligned}
$$

Suppose now that $x<\frac{\pi}{2 N}$. Then

$$
\sum_{n=2}^{N} \frac{1}{n \log n} \geq \sum_{n=2}^{N} \frac{\cos n x}{n \log n} \geq \cos N x \sum_{n=2}^{N} \frac{1}{n \log n} \geq\left(1-\frac{x^{2} N^{2}}{2}\right) \sum_{n=2}^{N} \frac{1}{n \log n} .
$$

Since

$$
\sum_{k=2}^{n} \frac{1}{k \log k}=\log \log n+B+O\left(\frac{1}{n \log n}\right)
$$

where $B$ is a constant (see Exercise 8.20 of [1]), we get for $x<\frac{\pi}{2 N}$,

$g(x)=\log \log N+B+O\left(\frac{1}{N \log N}\right)+O\left(x^{2} N^{2} \log \log N\right)+O\left(\frac{1}{x N \log N}\right)$

as $N \rightarrow \infty$.

Choosing $N=\frac{1}{x}\left(\log \frac{1}{x} \log \log \frac{1}{x}\right)^{-1 / 3}$, we obtain (4.6) as $x \rightarrow 0+$.

Remark 5 In the following example, for small values of $|x|$, we only need the following consequence of the preceding lemma:

$$
\sum_{n=2}^{\infty} \frac{\cos n x}{n \log n} \geq C \log \log (1 /|x|)
$$

which can also be obtained using Theorem V.1.5 of [19].

Example 2 Let $t_{0} \in(0,2 \pi)$. For $0<\delta<1$ define

$$
g(t)= \begin{cases}\pi / 2 & t_{0}-\delta<t<t_{0} \\ -\pi / 2 & t_{0}<t<t_{0}+\delta\end{cases}
$$


and suppose that $g$ is Hölder continuous elsewhere. By Theorem V.1.3 of [20], the series

$$
h(t)=2 \sum_{n=2}^{\infty} \frac{\sin n\left(t-t_{0}\right)}{n \log n}
$$

converges uniformly and defines a continuous function on $[0,2 \pi]$. It is well known that

$$
\tilde{h}(t)=-2 \sum_{n=2}^{\infty} \frac{\cos n\left(t-t_{0}\right)}{n \log n}
$$

which is continuous on $[0,2 \pi] \backslash\left\{t_{0}\right\}$ (see Theorem I.2.6 of [20]). Define $f=g+h$. Then $\lim _{t \rightarrow t_{0} \pm} f(t)=\mp \pi / 2$, so $f$ is piecewise continuous with only one jump, which is of size $\pi$. We want to determine whether $\exp (\tilde{f})$ is integrable. Obviously we cannot use Zygmund's Theorem A. Notice also that we cannot apply Theorem 1 because we do not know without further inspection whether $f \geq \pi / 2$ a.e. on $\left(0, t_{0}\right)$ and $f \leq-\pi / 2$ a.e. on $\left(t_{0}, 2 \pi\right)$.

Now, similarly to (1.6), and using the fact that $g$ is Hölder on $[0,2 \pi] \backslash\left\{t_{0}\right\}$, there is a constant $C>0$ such that

$$
\tilde{g}(t) \leq-\log \left|\sin \frac{t-t_{0}}{2}\right|+C
$$

for $0 \leq t \leq 2 \pi$. To estimate $\tilde{h}$ near $t_{0}$, notice first that Lemma 3 implies that

$$
\tilde{h}(t) \leq-2 \log \left(\log \left|t-t_{0}\right|^{-1}\right)
$$

for $t$ sufficiently close to $t_{0}$. Therefore, for some constants $C$, we have

$$
\begin{aligned}
e^{\tilde{f}(t)} & =e^{\tilde{g}(t)} e^{\tilde{h}(t)} \leq C \exp \left(-\log \left|\sin \frac{t-t_{0}}{2}\right|\right) \exp \left(-2 \log \log \frac{1}{\left|t-t_{0}\right|}\right) \\
& =C\left|\sin \frac{t-t_{0}}{2}\right|^{-1}\left(\log \frac{1}{\left|t-t_{0}\right|}\right)^{-2} \leq C\left|t-t_{0}\right|^{-1}\left(\log \left|t-t_{0}\right|\right)^{-2},
\end{aligned}
$$

which is integrable in a neighborhood of $t_{0}$, and hence $e^{\tilde{f}} \in L^{1}$.

Now, of course, by Theorem 1 , the integrability of $e^{\tilde{f}}$ means that $f$ has values in $(-\pi / 2, \pi / 2)$ on a set of positive measure. In fact, by V.2.13 of [19], the function $h$ is positive on $\left(t_{0}, t_{0}+\epsilon\right)$ for some $\epsilon>0$ and hence negative on $\left(t_{0}-\epsilon, t_{0}\right)$ (as an odd function), and so indeed $f<\pi / 2$ on $\left(t_{0}-\epsilon, t_{0}\right)$ and $f>-\pi / 2$ on $\left(t_{0}, t_{0}+\epsilon\right)$.

Remark 6 We can use the previous example to construct a function $f \in L^{\infty}$ such that $\|f\|_{\infty}=\pi / 2,|f|<\pi / 2$ and $e^{\tilde{f}} \in L^{1}$. This should be compared with Zygmund's result in (1.2). 
Open Problem 2 In Theorem 1, it is assumed that $f \in L^{\infty}$ as in Zygmund's Theorem A. It is natural to ask whether the conclusion of Theorem 1 remains true for unbounded functions.

We finish this section with a connection between the estimate in (1.8) and the distance in BMO to $L^{\infty}$ (see Sect. VI.6 of [4]).

Suppose that the conditions of Theorem 1 are satisfied, that is, $f \in L^{\infty}$ with $f \geq \pi / 2$ almost everywhere and $0<|E|<2 \pi$. If $\|g\|_{\infty}<\pi / 2$, then by Theorems A and $1, \widetilde{f \rho_{E}}-\tilde{g} \notin L^{\infty}$, and so $\operatorname{dist}\left(\widetilde{f \rho_{E}}, L^{\infty}\right) \geq \pi / 2$, where

$$
\operatorname{dist}\left(\varphi, L^{\infty}\right)=\inf \left\{\|g\|_{\infty}: \varphi-\tilde{g} \in L^{\infty}\right\}
$$

which is equivalent to $\inf _{g \in L^{\infty}}\|\varphi-g\|_{\mathrm{BMO}}$ (see, e.g., page 250 in [4]). By Corollary VI.6.6, there is no $\epsilon \in(0,1)$ such that

$$
\sup _{I} \frac{\left|\left\{t \in I: \widetilde{f \rho_{E}}(t)-\widetilde{\left(f \rho_{E}\right)_{I}} \mid>\lambda\right\}\right|}{|I|} \leq e^{-\lambda / \epsilon}
$$

for all $\lambda \geq 0$, where the supremum is taken over all arcs $I \subset \mathbb{T}$ and the average $\varphi_{I}$ is defined by $\varphi_{I}=\frac{1}{|I|} \int_{I} \varphi$ for $\varphi \in L^{1}$.

The same conclusion also follows directly from (1.8) if $f \geq \pi / 2$, which is no surprise because the requirement that $f$ has a gap in its essential range is a stronger assumption than $\operatorname{dist}\left(\widetilde{f \rho_{E}}, L^{\infty}\right) \geq \pi / 2$. Indeed, assume that (1.8) holds and (4.7) does not hold, so that there exists $\epsilon \in(0,1)$ satisfying the estimate (4.7). Denote $h=f \rho_{E}$ so that $\tilde{h}_{0}=\frac{1}{2 \pi} \int_{0}^{2 \pi} \tilde{h}$, and choose $s \geq 0$ so large that $\lambda=\tilde{h}_{0}+s \geq 0$. Then there exists a constant $C>0$ so that

$$
\begin{aligned}
C e^{-\lambda} & \leq \frac{1}{2 \pi}|\{\theta \in[0,2 \pi]: \tilde{h}(\theta)>\lambda\}|=\frac{1}{2 \pi}\left|\left\{\theta \in[0,2 \pi]: \tilde{h}(\theta)-\tilde{h_{0}}>s\right\}\right| \\
& \leq \sup _{I \subset \mathbb{T}} \frac{1}{|I|}\left|\left\{\theta \in I:\left|\left(\tilde{h}-\tilde{h}_{I}\right)(\theta)\right|>s\right\}\right| \leq e^{-\frac{s}{\epsilon}},
\end{aligned}
$$

so we have $C e^{-\lambda}=C e^{-\left(\tilde{h}_{0}+s\right)} \leq e^{-\frac{s}{\epsilon}}$. Therefore $C e^{-\tilde{h}_{0}} \leq e^{\left(1-\frac{1}{\epsilon}\right) s} \rightarrow 0$ as $s \rightarrow \infty$, which is a contradiction.

\section{Complex-Valued Functions}

While real-valued functions are of particular importance in the study of exponential integrability of their conjugate functions, especially in connection with applications, such as Riemann-Hilbert problems and spectral theory of Toeplitz operators above, it would also be of interest to consider the case of complex-valued functions. Indeed, as in Zygmund's Theorem A, we may consider a complex-valued $f \in L^{\infty}$ and ask under what conditions is $\exp \left(\mid \widetilde{f \rho_{E} \mid}\right)$ not integrable. As in the proof of Theorem 1 , we can define $m(\lambda)=\left|\left\{t: \exp \left(\left|\widetilde{f \rho_{E}}(t)\right|\right)>\lambda\right\}\right|$ and show that if there is a constant $\left|\left\{t: \widetilde{f \rho_{E}}(t) \mid>\lambda\right\}\right| \geq C e^{-\lambda}$ for all $\lambda \geq 0$, then $\exp \left(\widetilde{f \rho_{E} \mid}\right)$ is not integrable. 
However, in the complex case, the function $f \rho_{E}$ no longer has a similar (geometric) meaning as in the real case where it can be related to a gap in the essential range. For this reason, we say that a set $A \subset \mathbb{C}$ has a gap of size $g>0$ if $A=B \cup C$ for some sets $B$ and $C$ of positive measure with $\operatorname{dist}(A, B) \geq g$. With this, we can state (1.9) in Theorem 1 as follows: If $f \in L^{\infty}$ is real and $\mathcal{R}(f)$ has a gap of size at least $\pi$, then $\exp (\tilde{f})$ is not integrable.

Open Problem 3 Given a complex-valued function $f$ in $L^{\infty}$, find a converse to Zygmund's Theorem A.

It may be useful to try to relate the exponential integrability of $\tilde{f}$ to the size of the gap in the essential range of $f$ as in the real case.

Open Access This article is licensed under a Creative Commons Attribution 4.0 International License, which permits use, sharing, adaptation, distribution and reproduction in any medium or format, as long as you give appropriate credit to the original author(s) and the source, provide a link to the Creative Commons licence, and indicate if changes were made. The images or other third party material in this article are included in the article's Creative Commons licence, unless indicated otherwise in a credit line to the material. If material is not included in the article's Creative Commons licence and your intended use is not permitted by statutory regulation or exceeds the permitted use, you will need to obtain permission directly from the copyright holder. To view a copy of this licence, visit http://creativecommons.org/licenses/by/4.0/.

\section{References}

1. Apostol, T.M.: Mathematical Analysis, 2nd edn. Addison-Wesley Publishing Co., Reading (1974)

2. Axler, S., Bourdon, P., Ramey, W.: Harmonic Function Theory, vol. 137, 2nd edn. Springer, New York (2001)

3. Baernstein, A.: Analytic functions of bounded mean oscillation. In: Aspects of Contemporary Complex Analysis (Proc. NATO Adv. Study Inst., Univ. Durham, Durham, 1979), pp. 3-36. Academic Press, London-New York (1980)

4. Garnett, J.B.: Bounded Analytic Functions, Revised 1, p. 236. Springer, New York (2007)

5. Garnett, J.B., Marshall, D.E.: Harmonic Measure. Reprint of the 2005 original. New Mathematical Monographs, p. 2. Cambridge University Press, Cambridge (2008)

6. Goluzin, G.M.: Geometric Theory of Functions of a Complex Variable. Translations of Mathematical Monographs, vol. 26. American Mathematical Society, Providence, RI (1969)

7. Katznelson, Y.: An Introduction to Harmonic Analysis, 3rd edn. Cambridge University Press, Cambridge (2004)

8. Lavrent'ev, M. A., Shabat, B. V.: Methods of the Theory of Functions in a Complex Variable. (Russian; translated into French, German and Spanish) Fifth edition. Nauka, Moscow (1987).

9. Lekishvili, M.M.: Exponential integrability of a conjugate function. Math. Zametki (Russian) 48(2), 155-157 (1990)

10. Perälä, A., Virtanen, J.A., Wolf, L.: A Riemann-Hilbert problem with a vanishing coefficient and applications to Toeplitz operators. Concr. Oper. 1, 28-36 (2013)

11. Shargorodsky, E.: On some geometric conditions of Fredholmity of one-dimensional singular integral operators. Integral Equ. Oper. Theory 20(1), 119-123 (1994)

12. Shargorodsky, E.: A remark on the essential spectra of Toeplitz operators with bounded measurable coefficients. Integral Equ. Oper. Theory 57(1), 127-132 (2007)

13. Shargorodsky, E., Virtanen, J.A.: Uniqueness results for the Riemann-Hilbert problem with a vanishing coefficient. Integral Equ. Oper. Theory 56(1), 115-127 (2006)

14. Shargorodsky, E., Toland, J.F.: Bernoulli free-boundary problems. Mem. Am. Math. Soc. 196(914), 70 (2008)

15. Storoženko, È.A.: Embedding in the class $e^{L}$. Math. Zametki (Russian) 10, 17-24 (1971) 
16. Tsuji, M.: Potential Theory in Modern Function Theory. Reprinting of the 1959 Original. Chelsea Publishing Co., New York (1975)

17. Virtanen, J.A.: A remark on the Riemann-Hilbert problem with a vanishing coefficient. Math. Nachr. 266, 85-91 (2004)

18. Wolff, T.H.: Counterexamples to two variants of the Helson-Szegô theorem. Dedicated to the memory of Tom Wolff. J. Anal. Math. 88, 41-62 (2002)

19. Zygmund, A.: Sur les fonctions conjugées. Fund. Math. 13, 284-303 (1929)

20. Zygmund, A.: Trigonometric Series, vol. I, 3rd edn. Cambridge University Press, Cambridge (2002)

Publisher's Note Springer Nature remains neutral with regard to jurisdictional claims in published maps and institutional affiliations. 